Miel de abejas-Determinación del contenido de cenizas.

Miel y cera de abeja-Determinación de coliformes totales-Método de recuento en placa.

Anexo de Normas relacionadas con sistemas de calidad y gestión integral:

NCh2769.Of2003: Calificación de proveedores - requisitos generales.

NCh9000.Of2001: Sistemas de gestión de la calidad - Fundamentos y vocabulario.

NCh9004.Of2001: Sistemas de gestión de la calidad - Directrices para el mejoramiento del desempeño.

NCh-ISO10013.Of2003: Directrices para la documentación de sistemas de gestión de la calidad.

NCh-ISO14001.Of2005: Sistemas de gestión ambiental - Requisitos con orientación para su uso.
NCh-ISO14004.Of2005: Sistemas de gestión ambiental-Directrices generales sobre princi-

pios, sistemas y técnicas de apoyo.

NCh18000.Of2004:Sistemas de gestión - Prevención de riesgos

NCh18001.Of2004.:Sistemas de gestión - Prevención de riesgos

NCh18002.Of2004:Sistemas de gestión - Prevención de riesgos.

Directrices para la implementación de la norma NCh18001.

NCh-ISO19011.Of2003: Directrices para la auditoría de los sistemas de gestión de la calidad y/o ambiental.

NCh-ISO22000.c2006: Sistemas de gestión de la inocuidad de los alimentos - Requisitos para cualquier organización en la cadena alimentaria.

NCh-ISO22004.c2006: Sistemas de gestión de la inocuidad de los alimentos - Orientación para la aplicación de NCh-ISO 22000:2005.

Agro Sur 35(1): 6-10 2007

\title{
DIAGNÓSTICO TECNOLÓGICO: SISTEMAS DE ASEGURA- MIENTO DE CALIDAD PARA PYMES APÍCOLAS DE LAS REGIONES, LOS RÍOS Y LOS LAGOS.
}

\section{TECHNOLOGICAL DIAGNOSIS: SYSTEMS TO ENSURE QUALITY IN SMALL AND MEDIUM APICULTURE COMPANIES, FROM THE CHILEAN X AND XIV REGIONS.}

\author{
Alejandro Morán Villanueva \\ Nodo Apícola - Universidad Austral de Chile \\ Palabras clave: Aseguramiento de calidad, Pymes apícolas, nodo apícola.
}

La apicultura como rubro ha tomado importancia en los últimos años y para muchos productores ha pasado a ser su actividad económica principal. Con esto, los actores involucrados han notado la necesidad de entregar, adquirir y aplicar conocimientos tanto técnicos como de gestión, lo que ha llevado a que actividades relacionadas con capacitación en diversas áreas y el traspaso de conocimientos, cobren mayor interés.

El Ministerio de Agricultura a través de la Corporación Centro Apícola estableció en el año 2006 la Agenda Estratégica de la Cadena Apícola Nacional, entre cuyas líneas de acción se contempla la estandarización de los procesos productivos junto a la incorporación de sistemas de trazabilidad y la consolidación de una apicultura limpia y de calidad. Por estas razones, la entrega de conocimientos y la implementación de Buenas Prácticas para la Apicultura (BPA) y sistemas de aseguramiento de calidad certificables como las normas ISO y HACCP, es fundamental. En la búsqueda de cumplir con este objetivo la Universidad Austral 
de Chile, se encuentra ejecutando este año 2007, el proyecto denominado "Nodo de Difusión y Transferencia Tecnológica para PYMES Apícolas en Sistemas de Aseguramiento de Calidad", atendiendo a la convocatoria que realizó INNOVA-CHILE el año 2006.

El objetivo general del proyecto, es apoyar el mejoramiento productivo de PYMES apícolas a través de programas de difusión y transferencia tecnológica. Sus objetivos específicos son identificar necesidades tecnológicas de los apiarios, fortalecer capacidades para selección de equipos y tecnologías, entregar información especializada relativa a aseguramiento de calidad de procesos y productos apícolas y favorecer acceso a redes y fuentes de financiamiento públicos y privados para la modernización productiva e innovación.

El Nodo apoyará el mejoramiento productivo de las micro, pequeñas y medianas empresas apícolas, en especial aquellas cuya orientación productiva es la producción de miel para exportación, a través de programas de promoción de difusión y transferencia tecnológica. Se constituirá además en un nexo entre empresas de distinto tamaño dedicadas a la producción apícola. Con esto, se establecerá una red abierta a través de la cual podrán interactuar todos los actores involucrados en el rubro, teniendo acceso a información, capacitación, asistencia y a mejores tecnologías y prácticas disponibles en Chile y el resto del mundo.

Esta propuesta se centrará en aplicar conocimientos específicos a nivel sectorial relativos a sistemas de aseguramiento de calidad, apoyado por la experiencia adquirida por la Universidad Austral de Chile en trabajos en conjunto con empresas apícolas de diversas características, como son exportadoras de miel hasta empresas campesinas. Este trabajo estará enfocado principalmente a la difusión, capacitación e implementación de normas de aseguramiento de calidad ISO, NCh 2909 y BPA, con lo que se orientará los servicios de asistencia técnica a las PYMES, buscando cumplir con los objetivos propuestos.

Las actividades del proyecto comenzaron con la aplicación de un diagnóstico tecnológico, realizado entre el 25 de marzo y el 10 de abril en las provincias de Valdivia, Osorno y Llanquihue. El fin de éste, fue obtener una visión general a nivel de región y particular a nivel de 20 productores seleccionados. Permitió caracterizar a los apicultores en el ámbito de la implementación de sistemas de aseguramiento de calidad certificables.

El diagnóstico ha revelado principalmente que sobre el $65 \%$ de los productores tiene a la apicultura como fuente principal de ingresos. Esto concuerda con la tendencia de los últimos años, en la cual la apicultura deja de ser un rubro secundario y comienza a ser reconocida como una actividad productiva, en muchos casos, base de la economía familiar.

Entre las características de los productores diagnosticados, se puede apreciar que sobre un $70 \%$ cuenta con tres o más temporadas como apicultor. Esto puede indicar cierto grado de experiencia en la actividad que desarrolla y conocimiento de las labores que ella contempla.

La heterogeneidad en lo referente a las características de los productores se presenta en distintos ámbito, como el nivel de educación de los apicultores, ya que se encuentran apicultores en todos los niveles, predominando eso si los apicultores que cuentan con educación media completa. También se puede advertir esta diversidad, en el tamaño de las explotaciones y los volúmenes de miel que producen. Por un lado se pueden encontrar pequeños productores, que están incorporándose al rubro, los cuales cuentan con cinco colmenas y por otro lado, productores de mayor tamaño, ya consolidados con explotaciones que pueden superar las 1500. Se destaca el hecho de que aunque los productores cuentan con experiencia en el rubro, los apiarios generalmente son del perfil del pequeño productor.

Aunque las explotaciones apícolas son generalmente pequeñas, el mercado de destino de las mieles es bastante diverso, predominando el mercado regional y el exterior. La venta de la miel se desarrolla de distintas maneras, es decir, mediante canales formales e informales; directamente, a través de un intermediario o ambas a la vez. De esta forma se puede explicar el hecho de que los pequeños productores lleguen con sus mieles al exterior. Esta labor es facilitada por acopiadores como es APICOOP 
en la región, quien comercializa y distribuye las mieles en el extranjero. Es recomendable para realizar esta actividad que cada productor que destina su miel hacia el exterior, independiente del tamaño de su explotación, se encuentre en el Registro de Apicultores de Miel de Exportación (RAMEX). El $80 \%$ de los productores consultados cumple con este requisito. Sin embargo, como una fracción importante de los productores distribuye sus mieles a través de un intermediario como APICOOP, muchos no cuentan con resolución sanitaria del Servicio de Salud, y otra parte de ellos no cuenta con iniciación de actividades propia, no permitiéndoles actuar como microempresas independientes frente al sistema tributario nacional. Es importante regularizar esta situación, permitiendo a estos productores estar en un mismo nivel y contar con los mismos derechos y obligaciones que las empresas formalmente constituidas.

La producción de miel, como muchas otras actividades es un proceso, por lo tanto, cualquier irregularidad en alguna parte de esta cadena puede terminar en la generación de un producto que no sea llamativo para el cliente. Por lo tanto es necesario que los productores puedan controlar todos los aspectos de la línea de producción que estén a su alcance, para asegurar la calidad de estos. Si bien el 65\% de los productores señala conocer las características de sus proveedores, solo el $60 \%$ de estos puede respaldar la calidad de los productos que se les ofrece. Algunas medidas ya se han generado en esta área, las normas de BPA han establecido entre las labores necesarias para su certificación, la recopilación de datos y la implementación de registros entre los que se encuentran los proveedores. En este caso solo un $40 \%$ de los productores cuenta con un registro de estos, lo cual impide hacer un seguimiento de la cadena productiva, dificultando la trazabilidad de los productos.

La capacitación es otro factor importante en la producción. Se encuentra dada por la transmisión de técnicas y el aprendizaje de destrezas. En el área de sistemas de gestión, el 75\% de los apicultores diagnosticados ha asistido a algún curso de BPA, la mayoría realizados por la Universidad Austral de Chile en convenio con el Instituto de Desarrollo Agropecuario (INDAP). Esto representaría un conocimiento de estas reglas, las cuales son la base para otras de mayor exigencia. De los productores que han participado de capacitaciones en BPA, el 50\% reconoce haber implementado satisfactoriamente estas normas. Las principales necesidades se presentan al momento de buscar el financiamiento para dar cumplimiento a las exigencias que propone el programa, ya que en muchos casos, estos significan inversiones en infraestructura y variaciones en el manejo de los apiarios.

Otro aspecto importante es la asistencia técnica. El $80 \%$ de los productores encuestados no recibe asistencia técnica en este ámbito y el $20 \%$ restante, solo esporádicamente. Esta es una debilidad del rubro, ya que estos servicios tienen como fin divulgar los conocimientos que se generan en distintas materias de investigación y ponerlos a disposición de los productores. Entre las causas de que un grupo importante de apicultores no reciba este tipo de servicios, pueden ser que no existe una gran oferta de personal calificado para tratar y resolver estos problemas, un desconocimiento del servicio e incluso la falta de interés por parte de algunos por adquirirlo, principalmente por un tema de financiamiento.

En el ámbito del aseguramiento de calidad, solo un 35\% señala conocer algún sistema de gestión aparte de las BPA, entre las que destacan las normas ISO y las HACCP. Con esto, se advierte que se hace necesaria su difusión y sus requisitos para poder en primera instancia evaluar la pertinencia y factibilidad de su implementación. Esto se puede realizar a través de reuniones, charlas, visitas a planteles modelos, etc.

Relacionado con lo anterior, un muy bajo porcentaje (10\%) de los encuestados ha asistido a algún curso de capacitación en normas de aseguramiento de calidad certificables. Considerando la tendencia de los mercados, se puede reconocer como otra falencia del rubro y buscar una solución. La capacitación entrega a los apicultores las herramientas para autogestión, permitiéndoles ser más autosuficientes, ya que pueden hacerse cargo de la solución de algunos de sus problemas. Permite además a los productores advertir, cuales son los factores más 
importantes o los puntos críticos en el manejo de sus apiarios. A través de los años ha quedado demostrado que la capacitación presenta un impacto positivo sobre la producción.

El 40\% de los apicultores conoce algún requisito de los sistemas de aseguramiento de calidad, pero en estricto rigor se debe señalar que son conocidos superficialmente en la mayoría de los casos.. Existe en este punto una gran posibilidad de entregar información y transferir conocimientos con el fin de incrementar la eficiencia y competitividad de las empresas. Entre los apicultores que conocen algún sistema de gestión de calidad, solo el 5\% lo ha implementado. Hay que señalar que este proceso de implementación ha sido de carácter informal, por lo tanto, para cambiar esta situación, se hace necesaria la estandarización de estos procesos y la formación de recursos humanos capacitados para poder apoyar técnicamente, a los productores que deseen incorporarse. Esto demuestra que existe la clara necesidad de implementar estas normas de aseguramiento de calidad, con el fin de entregar al mercado un producto de calidad y que cuente con el componente inocuidad garantizado.

Un alto porcentaje de los apicultores (95\%) reconoce las ventajas de implementar algún sistema de gestión, enfocado al aseguramiento de calidad de los productos. Además, la totalidad de los productores, se ha planteado la necesidad de establecer procesos e incorporar recursos para mejorar sus productos, lo que muestra el interés que tienen los productores por modernizar sus procesos para estar más de acuerdo a las necesidades de los mercados. Sin embargo, menos de la mitad de los apicultores conoce algún instrumento de financiamiento para implementar alguno de estos sistemas. Entre las instituciones que ellos señalan como apoyo a este tipo de actividades se encuentran INDAP y CORFO. Esto muestra la necesidad, de difundir los distintos instrumentos que cada organismo pone a disposición de los usuarios. Un análisis de correspondencia múltiple, confirma que como características comunes entre los apicultores consultados, se encuentra por un lado la experiencia en el rubro, estar inscrito en el RAMEX y haber asistido a alguna capacitación en BPA y por otro, que no tienen resolución sanitaria del Servicio de Salud, no haber asistido a algún curso de capacitación en Normas ISO o NCh 2909 y no conocer las normas de aseguramiento de calidad certificables.

Estos resultados obtenidos generarán los lineamientos de las actividades a realizar con los apicultores entre las que destacan:

Auditorías a PYMES y explotaciones apícolas en sistemas de aseguramiento de calidad, con el fin de que puedan buscar la implementación de estas normas y trabajar como modelos. Se realizarán además visitas de seguimiento y asistencia técnica en la elaboración de un plan de trabajo para la implementación de sistemas de calidad y asesorías, para postulación a fondos para tales objetivos.

Difusión y Transferencia Tecnológica: Aparte del $7^{\circ}$ Encuentro Nacional de Ciencia y Tecnología Apícola, se organizarán durante el transcurso del año otras reuniones de difusión de resultados y experiencias adquiridas durante la ejecución del proyecto.

Capacitación: El Nodo Apícola contará además con un servicio de capacitación a distancia a través de una plataforma web, que fomentará el aprendizaje de los usuarios del proyecto en temas de aseguramiento de calidad y su implementación. Se realizarán cursos estructurados en cuatro módulos abiertos a la comunidad.

Misiones de captura tecnológica, que son visitas a empresas, universidades, centros de investigación o desarrollo tecnológico que sean de interés para la actividad. Considera además asistencia a seminarios, ferias o exposiciones de contenido tecnológico.

Consultorías especializadas realizadas por expertos en temas específicos con el fin de resolver problemas en el ámbito tanto tecnológico-productivo como en gestión. 